Published online 2017 April 13.

Abstract

\title{
Imaging oF Larynx and Hypopharynx
}

\section{Mohammad Ali Kazemi ${ }^{1, *}$}

${ }^{1}$ Assistant Professor of Tehran University of Medical Sciences, Amiraalam Hospital, Department of Radiology, Tehran Iran

"Corresponding author: Mohammad Ali Kazemi, MD, Assistant Professor of Tehran University of Medical Sciences, Amiraalam Hospital, Department of Radiology, Tehran Iran. E-mail: aliksp@yahoo.com

Received 2016 December 21; Accepted 2017 February 08.

\section{Abstract}

Squamous cell carcinoma comprises $95 \%$ of laryngeal carcinomas. It is usually detected by laryngoscopic evaluation. Imaging plays an important role in the evaluation of submucosal extension and staging of this malignancy. Supraglottic, glottic and sublottic regions are separately evaluated. In supraglottic region extension of the mass to preepilottic and paraglottic spaces and cartilges invasion are assssed. Bilateral TVCs and anterior commissure are evaluated in the glottic region and cricoid ring is scrutinized in subglottic area. Complete evaluation of cervical lymph nodes and distant metastasis are the next step. Hypopharyngeal SCC can involve pyriform sinus, post-cricoid and posterior pharyngeal walls. These must be differentiated from laryngeal carcinoma extension.

This is an abstract presented in the 33rd Iranian congress of radiology (ICR) and the 15th congress of Iranian radiographic science association (IRSA). 